\title{
About PANIC 2017
}

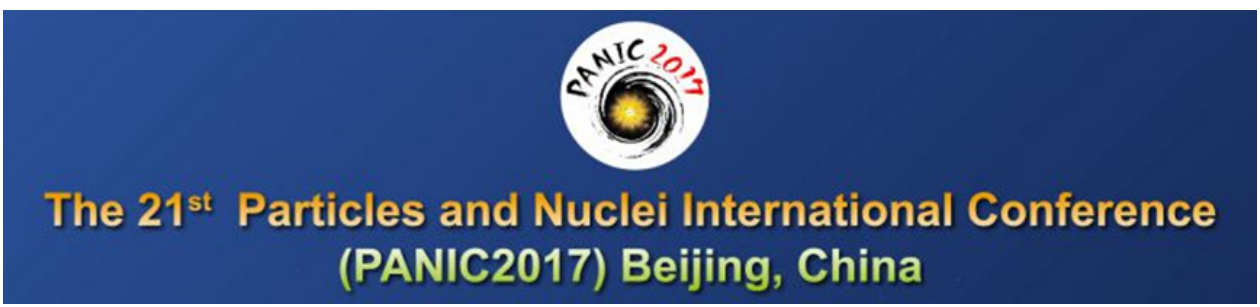

The 21st International Conference on Particles and Nuclei (PANIC 2017), organized by the Institute of High Energy Physics (IHEP), Chinese Academy of Sciences, has been held in Beijing from September 1 to 5, 2017. This conference is the 21st in the series of triennial conferences which bring together the Particle and Nuclear Physics communities.

The scientific program of PANIC addresses a broad range of topics at the interface between particle, nuclear and astrophysics. Special emphasis is devoted to recent discoveries and results. The program consists of plenary, parallel and poster sessions. Main topics include the following items:

- Hot and dense matter physics (QGP and heavy ion collision)

- Hadron spectroscopy and exotics

- Neutrino physics

- Dark matter and cosmology

- Nuclear and particle astrophysics

- Standard model physics at the TeV scale

- Energy frontier physics beyond the standard model

- Flavor physics - CKM and beyond

- Tests of symmetries and conservation laws

- Hadrons in medium - hyperons and mesons in nuclear matter

- Development of accelerators and detectors

- Applications of nuclear particle physics technology

This is an Open Access article published by World Scientific Publishing Company. It is distributed under the terms of the Creative Commons Attribution 4.0 (CC-BY) License. Further distribution of this work is permitted, provided the original work is properly cited. 


\section{Committees}

International Advisory Committee

Torsten Akesson, Univ. Lund, Sweden

Ursula Bassler, CNRS IN2P3, France

Reinhard Beck, Univ Bonn, Germany

Sergio Bertolucci, CERN, Switzerland

Martine Bosman, IFAE Barcelona, Spain

Philippe Chomaz, CEA, France

Jean Cleymans, Univ of Cape Town, South Africa

Umberto Dosselli, INFN, Italy

Paula Eerola, Univ of Helsinki, Finland

Fabiola Gianotti , CERN, Switzerland

Xiangdong Ji, SJTU, China

Ralf Bernd Kaiser, IAEA, Austria

Matthias Kasemann, DESY, Germany

Soo-Bong Kim, SNU, Korea

Young-Kee Kim, Univ. of Chicago, USA

Reiner Krücken, TRIUMF and UBC Vancouver,

Canada

David MacFarlane, SLAC, USA

Victor Matveev, JINR Dubna, Russia

Giora Mikenberg, Weizmann, Israel

Richard Milner, MIT, U.S.A

Joachim Mnich, DESY, Germany

Hugh E. Montgomery, JLAB, USA

Stephan Paul, TU Munich, Germany

Klaus Peters, GSI, Germany

Jianwei Qiu, JLAB, USA

Robert S Tschirhart, FNAL, USA

Yifang Wang, IHEP, China (Chair)

$\mathrm{Nu} \mathrm{Xu}, \mathrm{CCNU}$, China

Masanori Yamauchi, KEK, Japan

Bingsong Zou, ITP, China

\section{Local Organizing Committee}

Chair:

Yifang Wang, IHEP, China

Members:

Jun Cao, IHEP, China

Zhen Cao, IHEP, China

Lijun Guo, IHEP, China

Haibo Li, IHEP, China

Beijiang Liu, IHEP, China

Xinchou Lou, IHEP, China

Qian Pan, IHEP, China

Qing Qin, IHEP, China

Xiaoyan Shen, IHEP, China

Shuopin Wen,IHEP.China

Tianhong Xing,IHEP.China

Zhizhong Xing,IHEP.China

Changzheng Yuan, IHEP,

China

Xinmin Zhang, IHEP, China

Qiang Zhao, IHEP, China

Yangheng Zheng, UCAS,

China 


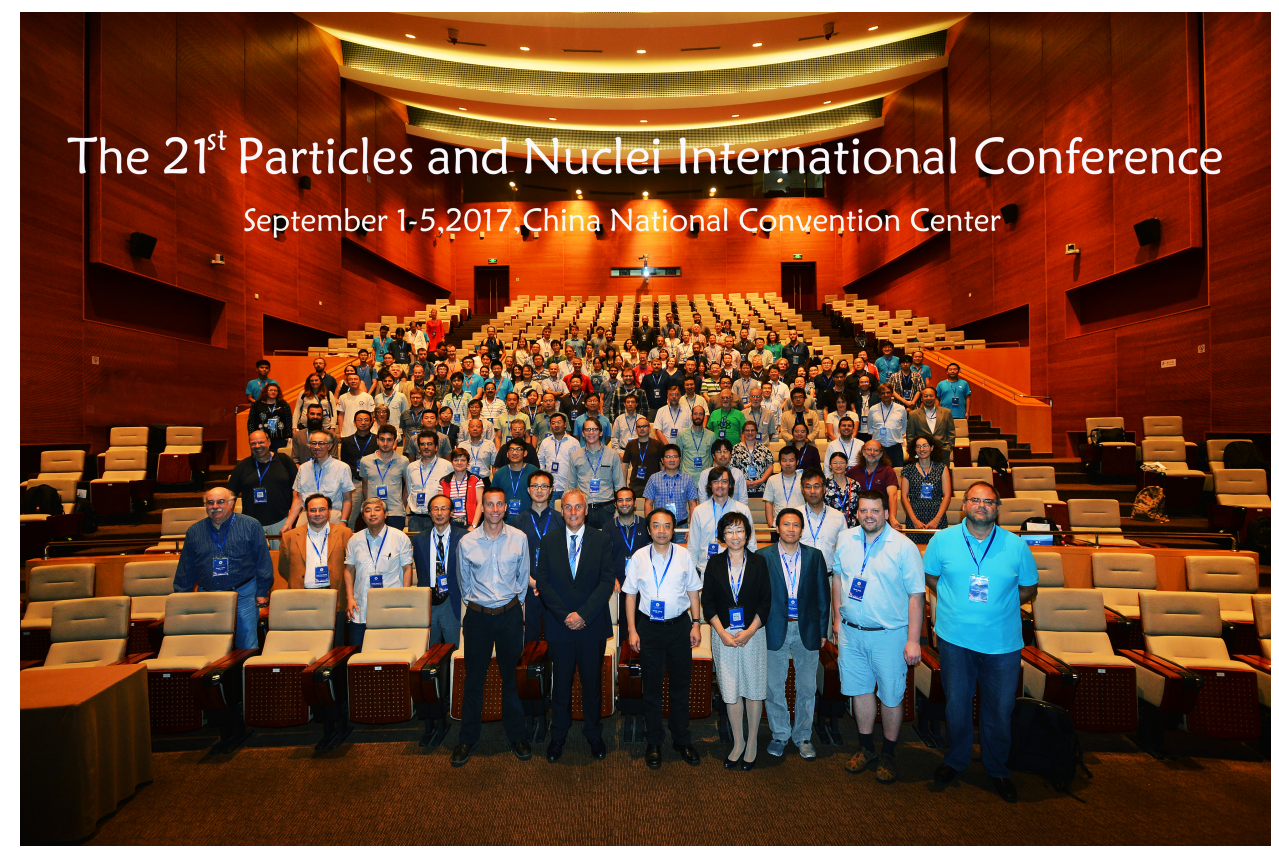

